\title{
CALCULATION OF DISCRETE SEMI-BOUNDED OPERATORS' EIGENVALUES WITH LARGE NUMBERS
}

\author{
S.I. Kadchenko', G.A. Zakirova ${ }^{2}$, L.S. Ryazanova', O.A. Torshina ${ }^{1}$ \\ ${ }^{1}$ Magnitogorsk State Technical University of G.I. Nosova, Magnitogorsk, Russian Federation \\ 2 South Ural State University, Chelyabinsk, Russian Federation \\ E-mail:kadchenko@masu.ru
}

In previous works of the article's authors on development of the Galerkin method, linear formulas for calculating the approximate eigenvalues of discrete lower semi-bounded operators have been obtained. The formulas allow calculating the eigenvalues of the specified operators of any number, regardless of whether the eigenvalues of the previous numbers are known or not. At that, it is possible to calculate the eigenvalues with large numbers when application of the Galerkin method is becoming difficult. It is shown that eigenvalues of small numbers of various boundary-value problems, generated by discrete lower semibounded operators and calculated by linear formulas and by the Galerkin method, are in a good conformity.

In this paper we use linear formulas to calculate approximate eigenvalues with large numbers of discrete lower semi-bounded operators. Results of calculation of eigenvalues by linear formulas and by known asymptotic formulas for two spectral problems are given. Comparison of the results of calculations of the approximate eigenvalues shows that they almost coincide for sufficiently large numbers. This proves the fact that linear formulas can be used for the considered spectral problems and sufficiently large numbers of eigenvalues.

Keywords: spectral problem; discrete operators; semi-bounded operators; eigenvalues and eigenfunctions of an operator; Galerkin method.

\section{Introduction}

It is known that the spectrum of a discrete operator consists of isolated points that have no limit points other than infinity. Moreover, each eigenvalue of a discrete operator has finite multiplicity.

Let $L$ be a discrete semi-bounded from below operator, defined in the separable Hilbert space $H$. Its eigenvalues $\mu$ are determined by finding non-trivial solutions of the equation:

$$
L u=\mu u,
$$

which satisfies the given homogeneous boundary conditions. Enumerate them in order of increasing values of eigenvalues, taking into account the multiplicity $\left\{\mu_{n}\right\}_{n=1}^{\infty}$.

To find the eigenvalues of the operator $L$ we use the Galerkin method. Consider a sequence $\left\{H_{n}\right\}_{n=1}^{\infty}$ of finite dimensional spaces $H_{n} \subseteq H$, which is complete in $H$. Suppose, that the orthonormal basis of space $H_{n}$ is known and consists of functions $\left\{\phi_{k}\right\}_{k=1}^{n}$. Wherein the functions $\phi_{k}$ must satisfy all boundary conditions of the problem. Following the Galerkin method, we will find the approximate solution of the spectral problem (1) in the form:

$$
u_{n}=\sum_{k=1}^{n} a_{k}(n) \phi_{k} .
$$

The following theorems were proved in [1].

Theorem 1. Let L be a discrete semi-bounded from below operator acting in a separable Hilbert space $H$. If the system of coordinate functions $\left\{\phi_{k}\right\}_{k=1}^{n}$ is a basis in the space $H$, then the Galerkin method applied to the problem of finding the eigenvalues of the spectral problem (1), constructed on this system of functions, converges.

Theorem 2. Let $L$ be a discrete semi-bounded from below operator acting in a separable Hilbert space $H$. If the system of coordinate functions $\left\{\phi_{k}\right\}_{k=1}^{n}$ is an orthonormal basis in the space H, then

$$
\tilde{\mu}_{n}(n)=\left(L \phi_{n}, \phi_{n}\right)+\delta_{n},
$$


where $\delta_{n}=\sum_{k=1}^{n-1}\left[\tilde{\mu}_{k}(n-1)-\tilde{\mu}_{k}(n)\right], \tilde{\mu}_{k}(n)$ is the Galerkin approximation of order $n$ to the corresponding eigenvalues $\mu_{k}$ of the operator $L$.

Formulas (3) allow, as shown in [1], to calculate the approximate eigenvalues of discrete semibounded operators with high computational efficiency. Unlike classical methods, they drastically reduce the amount of computation, solve the problem of finding the eigenvalues of any matrices of high order. Also formulas (3) allows to find eigenvalues regardless of whether know eigenvalues with lower numbers or not and solve the problem of calculating all necessary points of the spectrum of discrete semibounded operators.

Numerous eigenvalue calculations $\tilde{\mu}_{n}$ of boundary problems generated by discrete semi-bounded from below operators for $n \leq 50$ calculated by formulas (3) and the Galerkin method are in good agreement [1].

In this work, for further verification of the developed methodology for calculating the eigenvalues of discrete semi-bounded operators using formulas (3), we compare the results of their calculation using these formulas with the calculations using known asymptotic formulas for the following spectral problems.

\section{Asymptotic formulas for the eigenvalues of the spectral problems under consideration}

Consider the classical spectral problem of the form:

$$
-y^{\prime \prime}(x)+q(x) y(x)=\mu y(x), \quad \mu=\lambda^{2} \text { or } \mu=S^{2}, \quad 0<x<\pi
$$

with boundary conditions

$$
y(0)=0, \quad y(\pi)=0,
$$

or

$$
y(0)=0, \quad y^{\prime}(\pi)-h y(\pi)=0
$$

with the requirement that the potential $q(x)$, satisfying the condition:

$$
\int_{0}^{\pi} x|q(x)| d x<\infty
$$

In the thesis of Z.M. Gasimov [2] it was shown, that for eigenvalues $\mu_{n}$ of spectral problems (4), (5) and (4), (6) the following asymptotic formulas:

$$
\begin{gathered}
\mu_{n}=\lambda_{n}^{2}, \lambda_{n}=n+\frac{1}{\pi n} \int_{0}^{\pi} q(t) \sin ^{2}(n t) d t+O\left(r_{n}^{2)}\right) \\
\mu_{n}=S_{n}^{2}, S_{n}=n-0,5-\frac{h}{\pi(n-0,5)}+\frac{1}{\pi(n-0,5)} \int_{0}^{\pi} q(t) \sin ^{2}[(n-0,5) t] d t+O\left(\tilde{r}_{n}^{2)}\right)
\end{gathered}
$$

are true respectively. Here:

$$
\tilde{r}_{n}=\frac{1}{n}+r_{n}, \quad r_{n}=\int_{0}^{2 / n} t|q(t)| d t+\frac{1}{n} \int_{1 / 2 n}^{\pi}|q(t)| d t .
$$

To find the approximate eigenvalues of the spectral problem (4), (5) we construct a system of coordinate functions, each function of which is an eigenfunction of the spectral problem

$$
\begin{gathered}
-\phi^{\prime \prime}(x)=\beta \phi(x), \quad 0<x<\pi, \\
\phi(0)=0, \quad \phi(\pi)=0 .
\end{gathered}
$$

It is not difficult to show, that the spectral problem (9) has a set of eigenvalues $\left\{n^{2}\right\}_{n=1}^{\infty}$, which corresponds to an orthogonal system of eigenfunctions $\left\{C_{n} \sin (n x)\right\}_{n=1}^{\infty}$. Constants $C_{n}$ are found from the normalization conditions.

To find the approximate eigenvalues of the spectral problem (4), (6) we construct a system of coordinate functions, each function of which is an eigenfunction of the spectral problem: 


$$
\begin{gathered}
-\phi^{\prime \prime}(x)=\gamma \phi(x), \quad 0<x<\pi, \\
\phi(0)=0, \quad \phi^{\prime}(\pi)-h y(\pi)=0 .
\end{gathered}
$$

The set of eigenvalues $\left\{\gamma_{n}\right\}_{n=1}^{\infty}$ of the spectral problem (10) has no finite limit points. All the eigenvalues are real, non-negative, simple. They are the roots of the transcendental equation

$$
\sqrt{\gamma} \cos (\pi \sqrt{\gamma})-h \sin (\pi \sqrt{\gamma})=0
$$

and the corresponding system of eigenfunctions is orthogonal and have the form $\left\{C_{n} \sin \left(\sqrt{\gamma_{n}} x\right)\right\}_{n=1}^{\infty}$. Constants $C_{n}$ are found from the normalization conditions.

In case you need to find the eigenvalue $\gamma_{n}$ with a sufficiently large number it is difficult to use the transcendental equation (11), because it is necessary to consistently find all the values $\gamma_{n}$ with smaller numbers. This leads to a sharp increase in computational calculations. Therefore, in such cases it is necessary to use asymptotic formulas, which can be easily obtained from formulas (8), assuming that $q(t) \equiv 0$ :

$$
\gamma_{n}=S_{n}^{2}, S_{n}=n-0,5-\frac{h}{\pi(n-0,5)}+O\left(\tilde{r}_{n}^{2}\right), \tilde{r}_{n}=\frac{1}{n}
$$

\section{Numerical experiments}

Denote by $\tilde{\mu}$ the approximate eigenvalues of spectral problems (4), (5) and (4), (6), found by the Galerkin method, by $\hat{\mu}$ the eigenvalues found by formulas (3), by $\bar{\mu}$ the eigenvalues found by asymptotic formulas (7) or (8). In all the above calculations it was assumed that $\delta_{n}=0$.

In tables 1 and 2 the eigenvalues of problem (4), (5), found by formulas (3), and asymptotic formulas (7) with potential $q(x)=x^{2}-5 x+13-\sin (6 x)+2 e^{x}$ are given.

\begin{tabular}{|c|c|c|c|c|c|}
\hline$n$ & $\tilde{\mu}_{n}$ & $\hat{\mu}_{n}$ & $\bar{\mu}_{n}$ & $\left|\tilde{\mu}_{n}-\bar{\mu}_{n}\right|$ & $\left|\hat{\mu}_{n}-\bar{\mu}_{n}\right|$ \\
\hline 12 & 166,712 & 166,503 & 167,382 & $6,705 \cdot 10^{-1}$ & $8,792 \cdot 10^{-1}$ \\
\hline 13 & 191,685 & 191,507 & 192,257 & $5,719 \cdot 10^{-1}$ & $7,494 \cdot 10^{-1}$ \\
\hline 14 & 218,663 & 218,511 & 219,157 & $4,935 \cdot 10^{-1}$ & $6,463 \cdot 10^{-1}$ \\
\hline 15 & 247,646 & 247,513 & 248,076 & $4,302 \cdot 10^{-1}$ & $5,632 \cdot 10^{-1}$ \\
\hline 16 & 278,632 & 278,515 & 279,010 & $3,784 \cdot 10^{-1}$ & $4,951 \cdot 10^{-1}$ \\
\hline 17 & 311,620 & 311,517 & 311,956 & $3,354 \cdot 10^{-1}$ & $4,386 \cdot 10^{-1}$ \\
\hline 18 & 346,611 & 346,519 & 346,910 & $2,993 \cdot 10^{-1}$ & $3,913 \cdot 10^{-1}$ \\
\hline 19 & 383,602 & 383,520 & 383,871 & $2,687 \cdot 10^{-1}$ & $3,512 \cdot 10^{-1}$ \\
\hline 20 & 422,595 & 422,521 & 422,838 & $2,426 \cdot 10^{-1}$ & $3,170 \cdot 10^{-1}$ \\
\hline$\ldots$ & $\ldots$ & $\ldots$ & $\ldots$ & $\ldots$ & $\ldots$ \\
\hline 43 & 1871,545 & 1871,529 & 1871,598 & $5,261 \cdot 10^{-2}$ & $6,863 \cdot 10^{-2}$ \\
\hline 44 & 1958,544 & 1958,529 & 1958,595 & $5,025 \cdot 10^{-2}$ & $6,554 \cdot 10^{-2}$ \\
\hline 45 & 2047,544 & 2047,529 & 2047,592 & $4,804 \cdot 10^{-2}$ & $6,266 \cdot 10^{-2}$ \\
\hline 46 & 2138,543 & 2138,529 & 2138,589 & $4,597 \cdot 10^{-2}$ & $5,997 \cdot 10^{-2}$ \\
\hline 47 & 2231,543 & 2231,529 & 2231,587 & $4,404 \cdot 10^{-2}$ & $5,744 \cdot 10^{-2}$ \\
\hline 48 & 2326,542 & 2326,529 & 2326,584 & $4,222 \cdot 10^{-2}$ & $5,508 \cdot 10^{-2}$ \\
\hline 49 & 2423,542 & 2423,529 & 2423,582 & $4,052 \cdot 10^{-2}$ & $5,285 \cdot 10^{-2}$ \\
\hline 50 & 2522,541 & 2522,529 & 2522,580 & $3,892 \cdot 10^{-2}$ & $5,076 \cdot 10^{-2}$ \\
\hline 51 & 2623,541 & 2623,530 & 2623,578 & $3,740 \cdot 10^{-2}$ & $4,879 \cdot 10^{-2}$ \\
\hline$\ldots$ & $\ldots$ & $\ldots$ & $\ldots$ & $\ldots$ & $\ldots$ \\
\hline 63 & 3991,538 & 3991,530 & 3991,562 & $2,448 \cdot 10^{-2}$ & $3,197 \cdot 10^{-2}$ \\
\hline 64 & 4118,537 & 4118,530 & 4118,561 & $2,366 \cdot 10^{-2}$ & $3,098 \cdot 10^{-2}$ \\
\hline 65 & 4247,537 & 4247,530 & 4247,560 & $2,294 \cdot 10^{-2}$ & $3,004 \cdot 10^{-2}$ \\
\hline 66 & 4378,537 & 4378,530 & 4378,559 & $2,208 \cdot 10^{-2}$ & $2,913 \cdot 10^{-2}$ \\
\hline
\end{tabular}




\begin{tabular}{|c|c|c|c|c|c|}
\hline$n$ & $\tilde{\mu}_{n}$ & $\hat{\mu}_{n}$ & $\bar{\mu}_{n}$ & $\left|\tilde{\mu}_{n}-\bar{\mu}_{n}\right|$ & $\left|\hat{\mu}_{n}-\bar{\mu}_{n}\right|$ \\
\hline 67 & 4511,538 & 4511,530 & 4511,558 & $2,045 \cdot 10^{-2}$ & $2,827 \cdot 10^{-2}$ \\
\hline 68 & 4646,539 & 4646,530 & 4646,558 & $1,849 \cdot 10^{-2}$ & $2,744 \cdot 10^{-2}$ \\
\hline 69 & 4783,543 & 4783,530 & 4783,557 & $1,348 \cdot 10^{-2}$ & $2,665 \cdot 10^{-2}$ \\
\hline 70 & 4922,577 & 4922,530 & 4922,556 & $2,053 \cdot 10^{-2}$ & $2,590 \cdot 10^{-2}$ \\
\hline 71 & 5063,898 & 5063,530 & 5063,555 & $3,426 \cdot 10^{-1}$ & $2,517 \cdot 10^{-2}$ \\
\hline
\end{tabular}

Table 2

\begin{tabular}{|c|c|c|c|}
\hline$n$ & $\hat{\mu}_{n}$ & $\bar{\mu}_{n}$ & $\left|\hat{\mu}_{n}-\bar{\mu}_{n}\right|$ \\
\hline 1000 & 1000022,531 & 1000022,531 & $1,269 \cdot 10^{-4}$ \\
\hline 1001 & 1002023,531 & 1002023,531 & $1,267 \cdot 10^{-4}$ \\
\hline 1002 & 1004026,531 & 1004026,531 & $1,264 \cdot 10^{-4}$ \\
\hline 1003 & 1006031,531 & 1006031,531 & $1,262 \cdot 10^{-4}$ \\
\hline 1004 & 1008038,531 & 1008038,531 & $1,259 \cdot 10^{-4}$ \\
\hline$\ldots$ & $\ldots$ & $\ldots$ & $\ldots$ \\
\hline 10000 & 100000022,531 & 100000022,531 & $1,269 \cdot 10^{-6}$ \\
\hline 10001 & 100020023,531 & 100020023,531 & $1,267 \cdot 10^{-6}$ \\
\hline 10002 & 100040026,531 & 100040026,531 & $1,264 \cdot 10^{-6}$ \\
\hline 10003 & 100060031,531 & 100060031,531 & $1,268 \cdot 10^{-6}$ \\
\hline 10004 & 100080038,531 & 100080038,531 & $1,268 \cdot 10^{-6}$ \\
\hline$\ldots$ & $\ldots$ & $\ldots$ & $\ldots$ \\
\hline 100000 & 10000000022,531 & 10000000022531 & $1,269 \cdot 10^{-8}$ \\
\hline 100001 & 10000200023,531 & 10000200023,531 & $1,269 \cdot 10^{-8}$ \\
\hline 100002 & 10000400026,531 & 10000400026,531 & $1,269 \cdot 10^{-8}$ \\
\hline 100003 & 10000600031,531 & 10000600031,531 & $1,269 \cdot 10^{-8}$ \\
\hline 100004 & 10000800038,531 & 10000800038,531 & $1,269 \cdot 10^{-8}$ \\
\hline
\end{tabular}

Numerical calculations showed that the results of calculations of eigenvalues in three ways are in good agreement. As the number of eigenvalues increases, the difference between them decreases.

The results of calculations for sufficiently large numbers of the eigenvalues of the spectral problem (4), (5) are given in the table 2. The calculation of eigenvalues with such numbers by the Galerkin method causes difficulties due to the large dimensions of the matrices with which you have to work. Therefore, a comparison is made between the approximate eigenvalues found by formulas (3) and the asymptotic formulas (8). For $n>100000$ the values $\hat{\mu}_{n}$ and $\bar{\mu}_{n}$ are almost the same.

In Tables 3 and 4 the approximate eigenvalues of the spectral problem (4), (6) calculated by formulas (3) and asymptotic formulas (8) with $h=0,5$ and $q(x)=x^{3}-4 x+5-\cos (3 x)+e^{x}$ are given.

\begin{tabular}{|c|c|c|c|c|c|}
\hline \multicolumn{1}{|c|}{$\tilde{\mu}_{n}$} & $\hat{\mu}_{n}$ & $\bar{\mu}_{n}$ & $\left|\tilde{\mu}_{n}-\bar{\mu}_{n}\right|$ & $\left|\hat{\mu}_{n}-\bar{\mu}_{n}\right|$ \\
\hline 8 & 78,633 & 78,572 & 78,742 & $1,097 \cdot 10^{-1}$ & $1,740 \cdot 10^{-1}$ \\
\hline 9 & 96,619 & 96,572 & 96,708 & $8,864 \cdot 10^{-2}$ & $1,362 \cdot 10^{-1}$ \\
\hline 10 & 116,610 & 116,572 & 116,683 & $7,314 \cdot 10^{-2}$ & $1,114 \cdot 10^{-1}$ \\
\hline 11 & 138,603 & 138,572 & 138,665 & $6,137 \cdot 10^{-2}$ & $9,280 \cdot 10^{-2}$ \\
\hline 12 & 162,598 & 162,572 & 162,650 & $5,221 \cdot 10^{-2}$ & $7,849 \cdot 10^{-2}$ \\
\hline$\ldots$ & $\ldots$ & $\ldots$ & $\ldots$ & $\ldots$ & $\ldots$ \\
\hline 36 & 1338,574 & 1338,571 & 1338,581 & $6,287 \cdot 10^{-3}$ & $9,177 \cdot 10^{-3}$ \\
\hline 37 & 1412,574 & 1412,571 & 1412,580 & $5,957 \cdot 10^{-3}$ & $8,694 \cdot 10^{-4}$ \\
\hline 38 & 1488,574 & 1488,571 & 1488,580 & $5,653 \cdot 10^{-3}$ & $8,248 \cdot 10^{-4}$ \\
\hline 39 & 1566,574 & 1566,571 & 1566,579 & $5,371 \cdot 10^{-3}$ & $7,836 \cdot 10^{-4}$ \\
\hline 40 & 1646,574 & 1646,571 & 1646,579 & $5,110 \cdot 10^{-3}$ & $7,454 \cdot 10^{-3}$ \\
\hline$\ldots$ & $\ldots$ & $\ldots$ & $\ldots$ & $\ldots$ & $\ldots$ \\
\hline \hline
\end{tabular}


Математика

\begin{tabular}{|c|c|c|c|c|c|}
\hline$n$ & $\tilde{\mu}_{n}$ & $\hat{\mu}_{n}$ & $\bar{\mu}_{n}$ & $\left|\tilde{\mu}_{n}-\bar{\mu}_{n}\right|$ & $\left|\hat{\mu}_{n}-\bar{\mu}_{n}\right|$ \\
\hline 66 & 4428,572 & 4428,571 & 4428,574 & $1,771 \cdot 10^{-3}$ & $2,764 \cdot 10^{-3}$ \\
\hline 67 & 4562,573 & 4562,571 & 4562,574 & $1,561 \cdot 10^{-3}$ & $2,683 \cdot 10^{-3}$ \\
\hline 68 & 4698,573 & 4698,571 & 4698,574 & $1,104 \cdot 10^{-3}$ & $2,605 \cdot 10^{-3}$ \\
\hline 69 & 4836,577 & 4836,571 & 4836,574 & $3,285 \cdot 10^{-3}$ & $2,530 \cdot 10^{-3}$ \\
\hline 70 & 4976,588 & 4976,571 & 4976,574 & $1,422 \cdot 10^{-2}$ & $2,459 \cdot 10^{-3}$ \\
\hline
\end{tabular}

\begin{tabular}{|c|c|c|c|}
\hline \multicolumn{5}{|c|}{ Table 4 } \\
\hline$n$ & $\hat{\mu}_{n}$ & $\bar{\mu}_{n}$ & $\left|\hat{\mu}_{n}-\bar{\mu}_{n}\right|$ \\
\hline 1000 & 99906,571 & 999006,571 & $1,221 \cdot 10^{-5}$ \\
\hline 1001 & 1001006,571 & 1001006,571 & $1,219 \cdot 10^{-5}$ \\
\hline 1002 & 1003008,571 & 1003008,571 & $1,216 \cdot 10^{-5}$ \\
\hline 1003 & 1005012,571 & 1005012,571 & $1,214 \cdot 10^{-5}$ \\
\hline 1004 & 1007018,571 & 1007018,571 & $\ldots$ \\
\hline$\ldots$ & $\ldots$ & $\ldots$ & $1,220 \cdot 10^{-6}$ \\
\hline 10000 & 99990006,571 & 99990006,571 & $1,220 \cdot 10^{-6}$ \\
\hline 10001 & 100010006,571 & 100010006,571 & $1,219 \cdot 10^{-6}$ \\
\hline 10002 & 100030008,571 & 100030008,571 & $1,219 \cdot 10^{-6}$ \\
\hline 10003 & 100050012,571 & 100050012,571 & $\ldots$ \\
\hline 10004 & 100070018,571 & 100070018,571 & $1,220 \cdot 10^{-9}$ \\
\hline$\ldots$ & $\ldots$ & $\ldots$ & $1,220 \cdot 10^{-9}$ \\
\hline 100000 & 9999900006,571 & 9999900006,571 & $1,220 \cdot 10^{-9}$ \\
\hline 100001 & 10000100006,571 & 10000100006,571 & $1,220 \cdot 10^{-9}$ \\
\hline 100002 & 10000300008,571 & 10000300008,571 & $1,220 \cdot 10^{-9}$ \\
\hline 100003 & 10000500012,571 & 10000500012,571 & $1,219)$, \\
\hline 100004 & 10000700018,571 & 10000700018,571 & \\
\hline
\end{tabular}

The results of calculations of the approximate eigenvalues of the spectral problem (4), (6), given in Tables 3 and 4 are in good agreement.

\section{Conclusion}

Comparison of the results of calculations of the approximate eigenvalues of the spectral problems (4), (5) and (4), (6), carried out according to formulas (3) and asymptotic formulas (7) and (8), show that for sufficiently large numbers the results are almost the same.

In previous papers in the development of the Galerkin method linear formulas for calculating the approximate eigenvalues of discrete semibounded from below operators were obtained by the authors of the article. Formulas allow you to calculate the eigenvalues of the specified operators with any of their numbers, regardless of whether the eigenvalues with the previous numbers are known or not. In this case, it is possible to calculate the eigenvalues with large numbers, when the application of the Galerkin method becomes difficult. To test the new method for calculating the eigenvalues of discrete semibounded operators, computational experiments were conducted, which showed that the eigenvalues of small numbers of various boundary-value problems calculated by linear formulas and the Galerkin method are in good agreement. For further verification of the obtained linear formulas, it became necessary to find out how they behave when calculating eigenvalues with large numbers when asymptotic formulas begin to work. In this paper we use linear formulas to calculate approximate eigenvalues with large numbers of discrete semi-bounded from below operators. The results of calculating the eigenvalues by linear formulas and by known asymptotic formulas for two spectral problems are given. Comparison of the results of the calculations of the approximate eigenvalues show that for sufficiently large numbers they almost coincide. This confirms the fact that linear formulas can be used for the considered spectral problems and sufficiently large numbers of eigenvalues.

In the spectral problems considered, linear formulas give the same result as asymptotic formulas. This confirms the possibility of applying linear formulas to the approximate calculation of any eigenvalue of a discrete semi-bounded operator. By virtue of the linearity of formulas, finding eigenvalues becomes computationally efficient compared to any classical method. 
1. Kadchenko S.I., Zakirova G.A. A numerical method for inverse spectral problems. Bulletin of South Ural State University. Series of "Mathematical Modelling, Programming \& Computer Software", 2015, Vol. 8, no. 3, pp. 116-126. DOI: 10.14529/mmp150307

2. Gasymov Z.M. Reshenie obratnoy zadachi po dvum spektram dlya singulyarnogo uravneniya Shturma-Liuvillya: dis. ... kand. fiz.-mat. nauk (Solution of an inverse problem by two spectra for the singular Sturm-Liouville equation. Cand. phys. and math. sci. diss.), Baku, 1992, 121 p. (in Russ.).

Received December 10, 2018

Bulletin of the South Ural State University

Series "Mathematics. Mechanics. Physics" 2019, vol. 11, no. 1, pp. 10-15

УДК 519.642.8

DOI: 10.14529/mmph190102

\title{
ВЫЧИСЛЕНИЕ СОБСТВЕННЫХ ЗНАЧЕНИЙ С БОЛЬШИМИ НОМЕРАМИ ДИСКРЕТНЫХ ПОЛУОГРАНИЧЕННЫХ ОПЕРАТОРОВ
}

\author{
С.И. Кадченко ${ }^{1}$, Г.А. Закирова ${ }^{2}$, Л.С. Рязанова ${ }^{1}$, О.А. Торшина ${ }^{1}$ \\ ${ }^{1}$ Магнитогорский государственный технический университет, г. Магнитогорск, \\ Российская Федерация \\ ${ }^{2}$ Южно-Уральский государственный университет, г. Челябинск, Российская Федерация \\ E-mail:kadchenko@masu.ru
}

В предыдущих работах авторов статьи в развитии метода Галеркина получены линейные формулы для вычислений приближенных собственных значений дискретных полуограниченных снизу операторов. Формулы позволяют вычислять собственные значения указанных операторов любого номера независимо от того, известны ли собственные значения с предшествующими номерами или нет. При этом можно вычислять собственные значения и с большими номерами, когда применение метода Галеркина становится затруднительным. Показано, что собственные значения небольших номеров различных краевых задач, порожденных дискретными полуограниченными снизу операторами, вычисленные по линейным формулам и методом Галеркина, хорошо согласуются.

В работе применены линейные формулы для вычисления приближенных собственных значений с большими номерами дискретных полуограниченных снизу операторов. Приведены результаты вычислений собственных значений по линейным формулам и по известным асимптотическим формулам для двух спектральных задач. Сравнение результатов проведенных вычислений приближенных собственных значений показывает, что для достаточно больших номеров они практически совпадают. Это подтверждает тот факт, что для рассматриваемых спектральных задач и достаточно больших номеров собственных значений можно использовать линейные формулы.

Ключевые слова: спектральная задача; дискретные операторы; полуограниченные операторы; собственные числа и собственные функиии оператора; метод Галеркина.

\section{Литература}

1. Kadchenko, S.I. A numerical method for inverse spectral problems / S.I. Kadchenko, G.A. Zakirova // Вестник Южно-Уральского государственного университета. Серия «Математическое моделирование и программирование». - 2015. - Т. 8, № 3. - С. 116-126.

2. Гасымов, 3.М. Решение обратной задачи по двум спектрам для сингулярного уравнения Штурма-Лиувилля: дис. ... канд. физ.-мат. наук / 3.М. Гасымов. - Баку, 1992. - 121 с.

Поступила в редакцию 10 декабря 2018 г. 